\title{
Contribution of patchy reconnection to the ion-to-electron temperature ratio in the Earth's magnetotail
}

\author{
ChuXin Chen ${ }^{1 *}$, and Chih-Ping Wang ${ }^{2}$ \\ ${ }^{1}$ Chinese Academy of Sciences Key Laboratory of Geospace Environment, Department of Geophysics and Planetary Sciences, School of Earth and Space \\ Sciences, University of Science and Technology of China, Hefei 230026, China; \\ ${ }^{2}$ Department of Atmospheric and Oceanic Sciences, University of California, Los Angeles, CA, USA
}

\begin{abstract}
The ion-to-electron temperature ratio is a good indicator of the processes involved in the plasma sheet. Observations have suggested that patchy reconnection and the resulting earthward bursty bulk flows (BBFs) transport may be involved in causing the lower temperature ratios at smaller radial distances during southward IMF periods. In this paper, we estimate theoretically how a patchy magnetic reconnection electric field can accelerate ions and electrons differently. If both ions and electrons are non-adiabatically accelerated only once within each reconnection, the temperature ratio would be preserved. However, when reconnection occurs closer to the Earth where magnetic field lines are shorter, particles mirrored back from the ionosphere can cross the reconnection region more than once within one reconnection; and electrons, moving faster than ions, can have more crossings than do ions, leading to electrons being accelerated more than ions. Thus as particles are transported from tail to the near-Earth by BBFs through multiple reconnection, electrons should be accelerated by the reconnection electric field more times than are ions, which can explain the lower temperature ratios observed closer to the Earth.
\end{abstract}

Keywords: the ion-to-electron temperature ratio; plasma sheet; patchy magnetic reconnection; non-adiabatical acceleration

Citation: Chen, C. X., and Wang, C. P. (2019). Contribution of patchy reconnection to the ion-to-electron temperature ratio in the Earth's magnetotail. Earth Planet. Phys., 3(6), 474-480. http://doi.org/10.26464/epp2019049

\section{Introduction}

Among the parameters that are sensitive to the physical processes taking place in the plasma sheet, the ion-to-electron temperature ratio $\left(T_{\mathrm{i}} / T_{\mathrm{e}}\right)$ is one of the most important, and the variation of this ratio is affected by the mechanisms involved. Adiabatic processes would preserve the $T_{\mathrm{i}} / T_{\mathrm{e}}$ ratio (Wing et al., 2014). How non-adiabatic processes would change the $T_{\mathrm{i}} / T_{\mathrm{e}}$ ratio remains to be investigated.

Using THEMIS data, Wang CP et al. (2012) analyzed the dependence of the ion-to-electron temperature ratio on the local time and radial distance from the Earth, and found that the ratio $T_{\mathrm{i}} / T_{\mathrm{e}}$ decreases from $\sim 4$ to $\sim 2$ as $A E$ increases from 10 to 1000 . In addition, when the plasma sheet plasma is warm during a southward IMF period, comparison of the specific entropies between the distant- and near- Earth tail suggests that non-adiabatic processes energize the electrons more than the ions, which leads to a lower $T_{\mathrm{i}} / T_{\mathrm{e}}$ ratio at smaller radial distances. Earthward bursty bulk flows (BBFs), which can be generated by patchy reconnection (i.e., short time duration and short X-line length) in the plasma sheet, were observed to occur more frequently when $A E$ is higher (e.g., Wang CP et al., 2009). How ions and electrons are accelerated differently

Correspondence to: C. X. Chen, chuxin@ustc.edu.cn

Received 25 JUN 2019; Accepted 28 AUG 2019.

Accepted article online 12 SEP 2019.

(C) 2019 by Earth and Planetary Physics. by the reconnection electric field is not fully understood. Patchy reconnection also results in a plasma of reduced total entropy (plasma bubble) than its surrounding. This entropy difference moves the bubble plasma earthward with BBFs (Chen CX and Wolf, 1999) and can thus change the temperature ratio at smaller radial distances. It is therefore the intent of the present study to develop a theoretical estimation of how the $T_{\mathrm{i}} / T_{\mathrm{e}}$ ratio would be changed by a patchy magnetic reconnection electric field and how the resulting BBF transport would alter the radial profile of the ratio from midtail to the near-Earth.

This paper is organized as follows. In Section 2, we derive a set of equations describing the non-adiabatic acceleration of charged particle by a magnetic reconnection electric field. In Section 3, we calculate the $T_{\mathrm{i}} / T_{\mathrm{e}}$ ratios in the plasma sheet as a function of radial distances from $x=-60$ to $-10 R_{\mathrm{E}}$ using the derived equations and empirical magnetospheric plasma and magnetic field models. We compare the computed ratios with the observed ratios to evaluate the importance of the contribution from patchy reconnection. Finally, discussion and conclusion are provided in Section 4.

\section{Non-adiabatic Acceleration of Charged Particles by a}

\section{Patchy Magnetic Reconnection Electric Field}

Patchy magnetic reconnection may take place on the dayside magnetopause when the IMF is southward, since the typical duration of magnetic flux transfer events (FTEs) has been found to be approximately 1 to 2 min (Rijnbeek et al., 1984). When the IMF is 
northward, the duration of high-latitude reconnection is on the order of a couple of minutes (Onsager et al., 2001). Patchy magnetic reconnection may also take place at the nightside far tail as the ionospheric signature of magnetic reconnection, such as the auroral poleward boundary intensifications (PBIs), is short lived at 1-3 min (Zesta et al., 2002). Patchy reconnections may become frequent during substorms (Chen CX, 2016). In this section we investigate theoretically the changes in the ion-to-electron temperature ratio at the tail $\mathrm{X}$-line due to acceleration by patchy magnetic reconnection. Here we consider only the most essential reconnection element, that is, the reconnection electric field, and do not include other secondary elements, such as waves.

Figure 1 shows the left part of an X-line in the magnetotail. In the following explanation, we will focus in more detail on the upper half of this picture. In our calculations, the reconnection electric field is represented by $E$, the $x$-extension of the cross section of the flux tube is $\Delta x$, the X-line length is $\Delta y$ (Chen CX, 2013; Nakamura et al., 2004), the time duration of reconnection is $\Delta t$, the number density is $n$, the magnetic field is $B$, and the ion and electron temperatures in the pre-reconnected flux tube are $T_{\mathrm{i}}$ and $T_{\mathrm{e}}$, respectively.

Here we describe briefly the Speiser orbit in the current sheet (Speiser, 1965). When a charged particle from infinity enters a magnetic field reversal (the current sheet) with a small magnetic field perpendicular to the current sheet plane, it experiences two different types of motion. The first one is the gyration around the main anti-parallel magnetic field. Because of the different directions of the main fields above and below the current sheet, a particle on one side of the current sheet gyrating to the other side would experience a reversal in the direction of its gyromotion and would turn back; this causes the particle to be trapped within the current sheet plane with a meandering motion. But at the same time, the particle also experiences a second gyration around the small perpendicular magnetic field with a larger gyro-radius, and after completing half of this second gyromotion it is ejected from the current sheet and returns to infinity.

We assume that the $z$-extension of the domain of the non-adiabatic motion of the ions is on the order of ion gyroradius in the magnetic field $B$ due to the meandering motion around the neutral sheet in the $y z$-plane (Hill, 1975; Speiser, 1965), i.e.

$$
a_{\mathrm{i}}=\frac{m_{\mathrm{i}} \bar{U}_{\mathrm{i}}}{e B}
$$

where $m_{\mathrm{i}}$ is the mass of the proton, $\bar{U}_{\mathrm{i}}$ is the mean thermal speed of the proton, and $e$ is the electric charge of the electron. We further assume that the z-extension of the domain of the non-adiabatic motion of the electrons is on the order of electron gyroradius, i.e.

$$
a_{\mathrm{e}}=\frac{m_{\mathrm{e}} \bar{U}_{\mathrm{e}}}{e B}
$$

where $m_{\mathrm{e}}$ is the mass of electron, and $\bar{U}_{\mathrm{e}}$ is the mean thermal speed of electrons. Then total number of ions passing through the domain of the non-adiabatic motion of the ions should be

$$
\Delta N_{\mathrm{i}}=n \Delta x \Delta y a_{\mathrm{i}} \frac{\Delta t}{\tau_{\mathrm{i}}}
$$

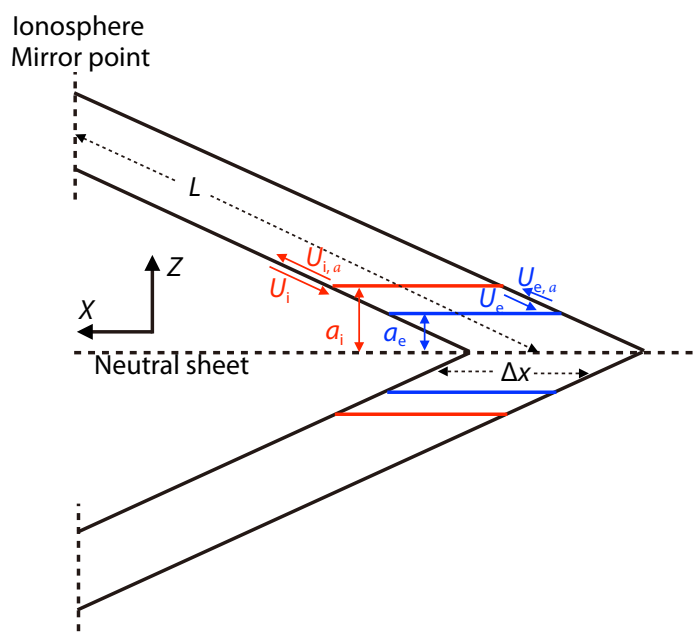

Figure 1. Sketch showing the left part of an X-line. The oblique magnetic field lines are in the $x z$-plane.

where

$$
\tau_{\mathrm{i}}=\frac{\pi r_{\mathrm{i}}}{\bar{U}_{\mathrm{i}}}
$$

is the time an ion will stay in the domain of the non-adiabatic motion of the ions, or the half ion cyclotron (in the $x y$-plane) period, and

$$
r_{\mathrm{i}}=\frac{m_{\mathrm{i}} \bar{U}_{\mathrm{i}}}{e B_{z}}
$$

is the ion gyroradius in the finite $z$-directional magnetic field $B_{z}$.

Substituting Equations (1), (4), and (5) into (3), we obtain

$$
\Delta N_{\mathrm{i}}=n \Delta x \Delta y \Delta t \frac{\bar{U}_{\mathrm{i}}}{\pi} \frac{B_{Z}}{B} .
$$

Likewise, the total number of electrons passing through the domain of the non-adiabatic motion of the electrons should be

$$
\Delta N_{\mathrm{e}}=n \Delta x \Delta y a_{\mathrm{e}} \frac{\Delta t}{\tau_{\mathrm{e}}}
$$

where

$$
\tau_{\mathrm{e}}=\frac{\pi r_{\mathrm{e}}}{\bar{U}_{\mathrm{e}}}
$$

is the time an electron will stay in the domain of the non-adiabatic motion of the electron or the half electron cyclotron (in the $x y$ plane) period, and

$$
r_{\mathrm{e}}=\frac{m_{\mathrm{e}} \bar{U}_{\mathrm{e}}}{e B_{z}}
$$

is the electron gyroradius in the finite $z$-directional magnetic field $B_{z}$. Substituting Equations (2), (8), and (9) into (7), we obtain:

$$
\Delta N_{\mathrm{e}}=n \Delta x \Delta y \Delta t \frac{\bar{U}_{\mathrm{e}}}{\pi} \frac{B_{z}}{B} .
$$

Although the particles have an equal chance to pass through the upper or lower portions of flux tube, we assume for simplicity that the particles move back to their original locations (the same argument applies to the lower part of the X-line). Each ion will have an energy increment through the domain of non-adiabatic motion of 


$$
\Delta w_{\mathrm{i}}=2 r_{\mathrm{i}} E e,
$$

and each electron will have an energy increment through the domain of non-adiabatic motion of

$$
\Delta w_{\mathrm{e}}=2 r_{\mathrm{e}} E e .
$$

The total energy increment of the ions through the domain of non-adiabatic motion will be

$$
\Delta W_{\mathrm{i}}=\Delta N_{\mathrm{i}} \Delta W_{\mathrm{i}}
$$

Substituting Equations (5), (6), and (11) into (13), we obtain

$$
\Delta W_{\mathrm{i}}=2 n \Delta x \Delta y \Delta t E \frac{m_{\mathrm{i}} \bar{U}_{\mathrm{i}}^{2}}{\pi B},
$$

where

$$
\bar{U}_{\mathrm{i}}=\sqrt{\frac{8 k T_{\mathrm{i}}}{\pi m_{\mathrm{i}}}},
$$

where $k$ is Boltzmann's constant.

Substituting Equation (15) into (14), we have

$$
\Delta W_{\mathrm{i}}=16 n \Delta x \Delta y \Delta t E \frac{k T_{\mathrm{i}}}{\pi^{2} B} .
$$

The total energy increment of the electrons through the domain of non-adiabatic motion would be

$$
\Delta W_{\mathrm{e}}=\Delta N_{\mathrm{e}} \Delta w_{\mathrm{e}}
$$

Substituting Equations (9), (10), and (12) into (17), we obtain

$$
\Delta W_{\mathrm{e}}=2 n \Delta x \Delta y \Delta t E \frac{m_{\mathrm{e}} \bar{U}_{\mathrm{e}}^{2}}{\pi B},
$$

where

$$
\bar{U}_{\mathrm{e}}=\sqrt{\frac{8 k T_{\mathrm{e}}}{\pi m_{\mathrm{e}}}} .
$$

Substituting Equation (19) into (18), we obtain

$$
\Delta W_{\mathrm{e}}=16 n \Delta x \Delta y \Delta t E \frac{k T_{\mathrm{e}}}{\pi^{2} B} .
$$

Let the total number of ions or electrons be $N$ in the upper part of the flux tube. Then, the total energy of the ions before reconnection is

$$
W_{\mathrm{i}}=\frac{3}{2} N k T_{\mathrm{i}}
$$

and the total energy of the electrons before reconnection is

$$
W_{\mathrm{e}}=\frac{3}{2} N k T_{\mathrm{e}}
$$

The ion-to-electron temperature ratio in the flux tube after reconnection will then be

$$
\frac{T_{\mathrm{i}, a}}{T_{\mathrm{e}, a}}=\frac{W_{\mathrm{i}}+\Delta W_{\mathrm{i}}}{W_{\mathrm{e}}+\Delta W_{\mathrm{e}}}=\frac{\frac{3}{2} N k T_{\mathrm{i}}+16 n \Delta x \Delta y \Delta t E \frac{k T_{\mathrm{i}}}{\pi^{2} B}}{\frac{3}{2} N k T_{\mathrm{e}}+16 n \Delta x \Delta y \Delta t E \frac{k T_{\mathrm{e}}}{\pi^{2} B}}=\frac{T_{\mathrm{i}}}{T_{\mathrm{e}}},
$$

where the subscript $a$ represents the time after reconnection.

Equation (23) indicates that, despite the increase of both $T_{\mathrm{i}}$ and $T_{\mathrm{e}}$ after the ions and electrons pass through a patchy magnetic re- connection, the ratio $T_{\mathrm{i}} / T_{\mathrm{e}}$ remains the same. However, this holds only when the ions and electrons pass through the reconnection domain a single time. As shown in Figure 1, when these particles are mirrored back from the ionosphere, they can actually pass through the same domain of non-adiabatic motion more than once within the $\Delta t$. In that case, Equation (16) becomes

$\Delta W_{\mathrm{i}}=16 n \Delta x \Delta y E \frac{k}{\pi^{2} B}\left(T_{\mathrm{i}} \tau_{\mathrm{i}}+T_{\mathrm{i}} t_{\text {i, roundtrip }, 1}+T_{\mathrm{i}, 1} T_{\mathrm{i}}+T_{\mathrm{i}, 1} t_{\mathrm{i}, \text { roundtrip }, 2}+\ldots\right)$,

where

$$
t_{\mathrm{i}, \text { roundtrip }, I}=2 \frac{L}{\bar{U}_{\mathrm{i}, I}}
$$

is the round trip traveling time of the ions in the distance $L$ from the reconnection site to the mirror point and

$$
\bar{U}_{\mathrm{i}, I}=\sqrt{\frac{8 k T_{\mathrm{i}, I}}{\pi m_{\mathrm{i}}}},
$$

where

$$
T_{\mathrm{i}, I}=T_{\mathrm{i},(I-1)}+\frac{4 r_{\mathrm{i},(I-1)} E e}{3 k},
$$

and the subscript / represents the /th repetition of non-adiabatic acceleration.

Likewise, Equation (20) becomes

$$
\begin{aligned}
\Delta W_{\mathrm{e}}= & 16 n \Delta x \Delta y E \frac{k}{\pi^{2} B} \\
& \times\left(T_{\mathrm{e}} \tau_{\mathrm{e}}+T_{\mathrm{e}} t_{\mathrm{e}, \text { roundtrip }, 1}+T_{\mathrm{e}, 1} \tau_{\mathrm{e}}+T_{\mathrm{e}, 1} t_{\mathrm{e}, \text { roundtrip }, 2}+\ldots\right),
\end{aligned}
$$

where

$$
t_{\mathrm{e}, \text { roundtrip }, I}=2 \frac{L}{\bar{U}_{\mathrm{e}, I}}
$$

is the round trip time of the electron and

$$
\bar{U}_{\mathrm{e}, I}=\sqrt{\frac{8 k T_{\mathrm{e}, I}}{\pi m_{\mathrm{e}}}},
$$

where

$$
T_{\mathrm{e}, I}=T_{\mathrm{e},(I-1)}+\frac{4 r_{\mathrm{e},(I-1)} E e}{3 k}
$$

Then the ion-to-electron temperature ratio will be

$$
\frac{T_{\mathrm{i}, a}}{T_{\mathrm{e}, a}}=\frac{W_{\mathrm{i}}+\Delta W_{\mathrm{i}}}{W_{\mathrm{e}}+\Delta W_{\mathrm{e}}} \neq \frac{T_{\mathrm{i}}}{T_{\mathrm{e}}} \text {. }
$$

It is noteworthy that because the number density $n$, the $x$-extension of the cross section of the flux tube $\Delta x$, and the X-line length $\Delta y$, all appear both explicitly or implicitly in the numerator and denominator of Equations (23) and (32), the ion-to-electron temperature ratio calculated by these two equations are independent of the quantities $n, \Delta x$ and $\Delta y$.

\section{Variation of Temperature Ratio from Midtail to Near- Earth Tail}

To estimate quantitatively how the acceleration by a patchy tail 
reconnection electric field and the resulting earthward BBF transport will alter the radial profile of the ion-to-electron temperature ratio in the plasma sheet, we compute Equation (32) by using two empirical magnetospheric models. The Tsyganenko T96 magnetospheric magnetic field model (Tsyganenko, 1995) and Tsyganenko tail plasma sheet model (Tsyganenko and Mukai, 2003) are used for magnetic field and plasma, respectively, where the input parameters are: a solar wind density of $6 \mathrm{~cm}^{-3}$, velocity of 380 $\mathrm{km} / \mathrm{s}, \mathrm{IMF} B_{z}=-5 \mathrm{nT}$, and Dst $=-80 \mathrm{nT}$.

Figure 2 shows schematically the procedure of our calculation. From the T96 magnetic field model, an equatorial point $x_{1}$ is first located. Then the magnetic field line passing through this point is traced out to the ionosphere, and the length $2 L$ of this field line is recorded. The plasma data on this field line are determined from the Tsyganenko tail plasma sheet model.

Assuming that a patchy magnetic reconnection occurs at the midpoint $x_{\text {half }}$ of this field line (Borovsky et al., 1998), the cut flux tube moves earthward as bursty bulk flows. The total entropy $(S)$ of the earthward half of the cut flux tube (indicated by $S_{\text {cut-tube }}$ shown in Figure 2) is calculated and compared with the entropies of background plasma (indicated by $S_{\text {background }}$ in Figure 2) on the equatorial plane at the same $y$ value. When $S_{\text {cut-tube }}$ is lower than the $S_{\text {background }}$ at $x_{\text {half, }}$, the flux tube moves earthward as a BBF due to interchange motion, and its earthward motion will stop at a final position, $x_{\text {final }}$, where $S_{\text {cut-tube }}=S_{\text {background }}$ at $x_{\text {final }}$ (Chen CX and Wolf, 1999). In our calculation, we bear in mind that BBFs can be responsible for $>60 \%$ of earthward transport of mass and energy when $A E$ is high (Angelopoulos et al., 1994), and BBFs are produced by magnetic reconnection. In this simplified picture, for plasma to be transported from the far tail to the near-Earth tail,
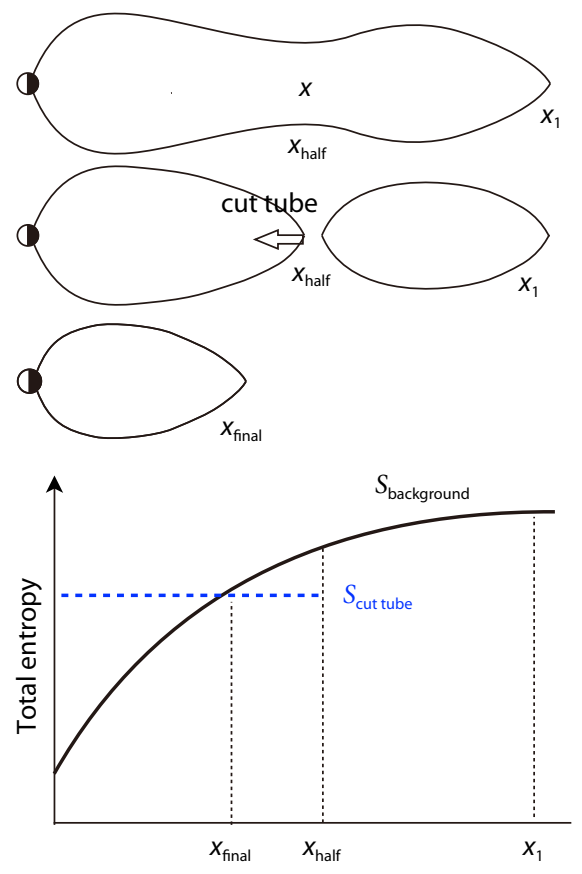

Figure 2. Sketch showing patchy magnetic reconnection and related positions. A flux tube whose $T_{\mathrm{i}} / T_{\mathrm{e}}$ ratio is under investigation, originally extending to $x_{1}\left(x_{1}=2 x_{\text {half }}\right)$, is cut by a reconnection taking place at $X_{\text {half }}$ having its final position at $x_{\text {final }}$. magnetic reconnection on the same flux tube is required. For example, a flux tube extending to $x=-48$ and $y=0$ is cut by a reconnection at $x=-24$ and $y=0$ and will then move to its final position at $x=-10.2$ and $y=0$. Only when the total entropy of a flux tube is reduced, is it possible for a cut flux tube to move earthward. The friction or turbulence heating effect of BBF is not considered in the present study.

Figure 3 shows one of our calculations. In this run, for the reconnection the electric field $E$ was set to $4 \mathrm{mV} / \mathrm{m}$, the time duration of reconnection $\Delta t$ was set to $60 \mathrm{~s}$, and the $z$-directional magnetic field $B_{z}$ was set to $0.5 \mathrm{nT}$. As can be seen from the equations in Section 2, these quantities are not independent of each other. We also experimented with other values of $E$ from $4 \mathrm{mV} / \mathrm{m}$ to $10 \mathrm{mV} / \mathrm{m}, \Delta t$ from $40 \mathrm{~s}$ to $80 \mathrm{~s}$, and $B_{z}$ from $0.5 \mathrm{nT}$ to $1.0 \mathrm{nT}$; the results were similar.

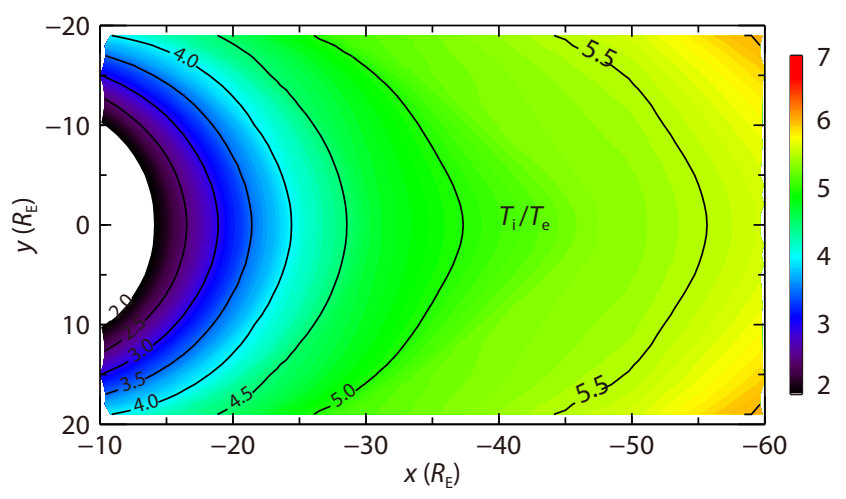

Figure 3. Contours of the calculated ion-to-electron temperature ratio on the equatorial plane.

The domain of our calculation $\left(x=-10\right.$ to $-60 R_{\mathrm{E}}$ and $y=-20$ to $20 R_{\mathrm{E}}$ ) is limited by the domain of the Tsyganenko tail plasma sheet model, which is valid for $10-50 R_{\mathrm{E}}$ down the Earth tail. We linearly interpolated the equatorial plasma data between $50 R_{E}$ (the tailward boundary of Tsyganenko tail plasma sheet model) and $220 R_{\mathrm{E}}$ down the tail, with the observed plasma number density of $0.3 \mathrm{~cm}^{-3}$ and an electron temperature of $1.2 \times 10^{6} \mathrm{~K}$ at $220 R_{\mathrm{E}}$ (Slavin et al., 1985).

The magnetic field strength at the mid-point of each pre-reconnected field line is represented as $B$ in the calculation. The electron temperature was set to be six times smaller than the ion temperature (i.e., close to the ratio in the magnetosheath) in each prereconnected field line.

There are several steps to produce the results shown in Figure 3. Step 1, the domain ( $x$ from -10 to $-60 R_{\mathrm{E}}$ and $y$ from -20 to $20 R_{\mathrm{E}}$ ) and the grid spacing $\left(1 R_{\mathrm{E}}\right)$ are determined. Step 2 , the volumes of unit flux tube are calculated from the Tsyganenko T96 magnetic field model ( $x$ from -10 to $-220 R_{\mathrm{E}}$ and $y$ from -20 to $20 R_{\mathrm{E}}$ ). Step 3, the plasma pressure is obtained from the Tsyganenko tail plasma sheet model ( $x$ from -10 to $-220 R_{\mathrm{E}}$ and $y$ from -20 to $20 R_{\mathrm{E}}$ ) and, with the above flux tube volume, the entropies of unit flux tube are estimated. Step 4, the entropies of half-way cut flux tube at $x_{\text {half }}$ are estimated ( $x$ from -10 to $-220 R_{\mathrm{E}}$ and $y$ from -20 to $20 R_{\mathrm{E}}$ ). Step 5, the half-way cut flux tube moves earthward and its stop 
location, $x_{\text {final, }}$ where the entropy of the half-way cut flux tube equals to the background plasma, is estimated. $x_{\text {final }}$ is limited to domain ( $x$ from -10 to $-60 R_{\mathrm{E}}$ and $y$ from -20 to $20 R_{\mathrm{E}}$ ). Step 6, Equation (32) is used to calculate the temperature ratio at each $x_{\text {half }}$ point and the value of the ratio is assigned to $x_{\text {final }}$ point.

The calculated ion-to-electron temperature ratios in the equatorial plane shown in Figure 3 are comparable to observational values shown in the left (warm plasma) and right panels (cool and warm combined) of Figure 4 (the observed asymmetry between dawn and dusk is not considered in the present theoretic work). In particular, the closer a position is to the Earth, the lower is the ratio. Note that our theoretical values of $T_{\mathrm{i}} / T_{\mathrm{e}}$ differ from the observed values. We attribute the lack of perfect agreement to effects we did not consider in our simplified model. The ratio is slightly higher toward dawn or dusk than at midnight. It is noteworthy that the $T_{\mathrm{i}} / T_{\mathrm{e}}$ ratio is preserved tailward of $60 R_{\mathrm{E}}$ because of the large distance between the reconnection site and the ionosphere. This comparison thus suggests that patchy magnetic reconnection electric fields and the resulting BBF transports are likely to be the main contributor to the observed radial variation in the temperature ratio during southward IMF periods.

\section{Discussion and Conclusion}

Our study has demonstrated that patchy tail reconnection electric fields and the resulting BBF transports can be important to radial variation of the ion-to-electron temperature ratio from the midtail to the near-Earth tail during southward IMF periods.

The point of view of the present study is microscopic. In our formula, the temperature (a macroscopic quantity) ratio has meaning only when convective flow or bulk velocity drops to zero. The temperature ratio estimated or measured in the exhaust of a reconnection is not the same as that when fast flows come to rest near the Earth. Therefore, the detailed processes that control the fraction of energy distributed between bulk flow energy and thermal energy are not relevant in present description. We assume that all kinetic energy gain by particles from the reconnection electric field will finally be converted into thermal energy when plasma is settled down in the near Earth plasma sheet.

A significant difference between the present study and those of Onsager et al. (1991) and Schriver et al. (1998) is our consideration of the electromagnetic field experienced by charged particles in the plasma sheet. In our study, the electric field was not assumed to be constant and steady, but was enhanced in some local channels (with varying length from the Earth) and short lived. The acceleration of charged particles in such a spatially and temporally short electric field is fundamentally different from that under a uniform and steady one. Besides the electric field, the magnetic field experienced by charged particles is also very different. After leaving the reconnection site, a flux tube is dipolarized, in contrast to the steady one. The motion of a charged particle in such dipolarized field lines is adiabatic. The reconnection sites are not necessarily confined only to the far tail; it is possible that they can be anywhere in the plasma sheet.

Our theoretical estimate of $T_{\mathrm{i}} / T_{\mathrm{e}}$ has been constructed to explain the statistical observation results; thus we use the Tsyganenko empirical magnetic field model in our calculation. The equatorial magnetic field after reconnection is assumed to be more dipolar (associated with BBFs and DF) than the background field lines. Values for the two large-scale magnetic field quantities used in our theoretical calculation - field-line length and flux tube volume - are determined primarily by the distance from the equatorial location to its ionosphere. Thus, even though local magnetic field changes near the equator after reconnection should affect the two quantities, the effects are not expected to be substantial. Therefore, despite the time independence of the Tsyganenko magnetic field, and without reconnection built in, it can empirically provide us with reasonable estimates of these two quantities: the magnetic fields associated both with a reconnected flux tube and with the background plasma sheet.

The location of a reconnection site, or more precisely the distance from a reconnection site to the ionosphere, plays a crucial role in the preservation or variation of the ion-to-electron temperature ratio. The non-preservation of the $T_{\mathrm{i}} / T_{\mathrm{e}}$ ratio comes from terms higher than the second one on the righthand side of Equations
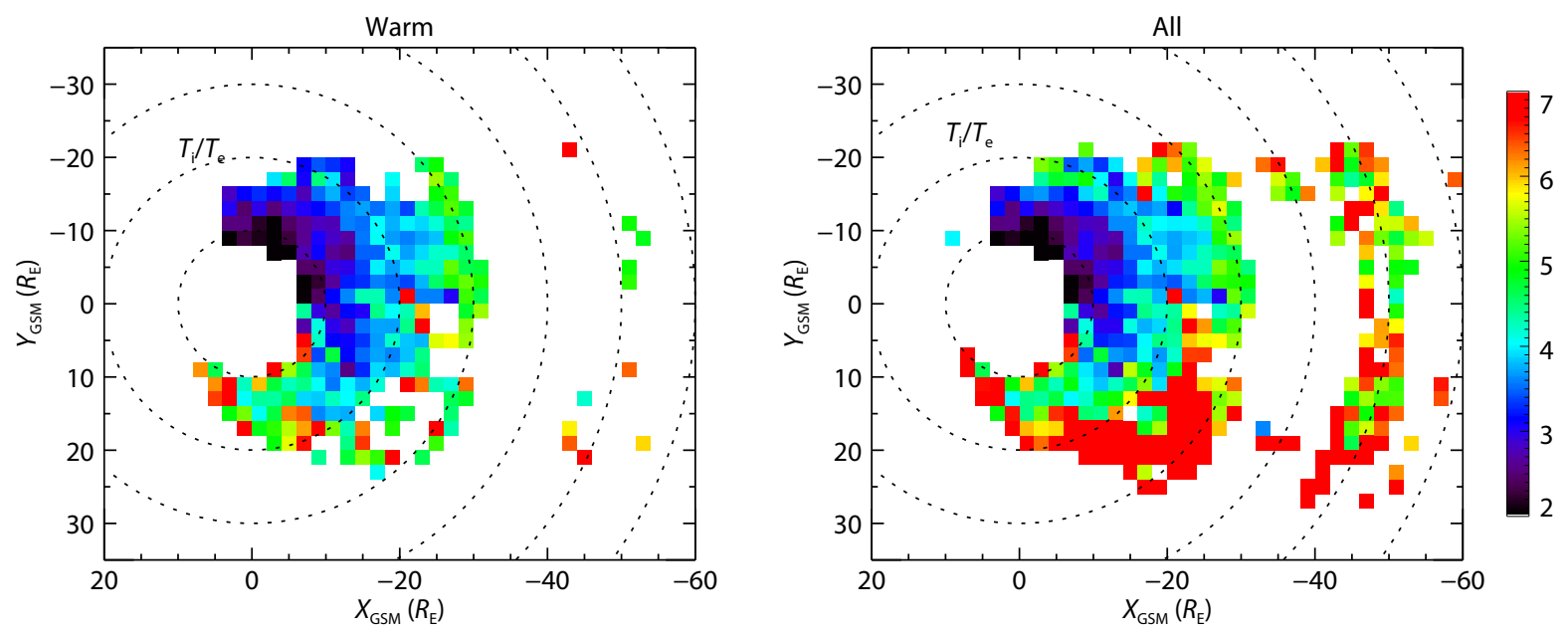

Figure 4. The observed $T_{\mathrm{i}} / T_{\mathrm{e}}$ ratio in the plasma sheet on the $X-Y$ plane. The left panel is for warm plasma, and the right panel is for the combination of cool and warm plasma. 
(24) and (28). In other words, when the effective time duration of reconnection is less than the sum of the half cyclotron period and the round trip travel time from the reconnection site to the ionosphere for both the accelerated ions and electrons, the ratio is preserved. The much lighter electron mass compared to that of an ion results in cyclotron periods far lower for electrons than for ions, as can be seen from Equations (4) and (8). For the observed $T_{\mathrm{i}} / T_{\mathrm{e}}$ ratio in the magnetosphere, electrons have a much higher thermal speed than ions. Consequently, the round trip travel time from the reconnection site to the ionosphere and back is much less for electrons than it is for ions, as can be seen from Equations (25) and (29). In the region earthward of $60 R_{E}$, ions experience non-adiabatic acceleration no more than once, while most electrons experience non-adiabatic acceleration more than once, leading to a lower $T_{\mathrm{i}} / T_{\mathrm{e}}$ ratio than is observed in the magnetosheath.

The ionosphere could be an energy sink for magnetospheric particles. For the study of the ion-to-electron temperature ratio, which requires fractional particle exchange between the magnetosphere and the ionosphere at short time scales (tens of minutes), this effect is negligible, because the number flux of precipitation particles is too small to accomplish this task. For example, taking the precipitation number flux as $7.93 \times 10^{6}$ $\mathrm{cm}^{-2} \cdot \mathrm{sec}^{-1} \cdot \mathrm{sr}^{-1}$ (Hardy et al., 1989), a flux tube extending to $20 R_{\mathrm{E}}$ down tail with a number density of $0.3 \mathrm{~cm}^{-3}$ will take about 140 hours to be replaced. Thus in the present investigation the ionosphere has been treated only as a reflector of magnetospheric particles.

Auroral electron acceleration is not considered in our model; it is driven by the parallel electric field that is provided by the plasma (inner source) within the reconnecting flux tube. The combination of convergent geometry of magnetic field lines on the ionosphere and the different gyroradius of ion and electron yields the parallel electric field. Such energy transfer from ions to electrons (self-adjustment of the plasma) should be minor compared to the whole energy of the plasma within a flux tube, because it is basically a boundary effect of the flux tube. In other words, only a very small fraction of electrons in the plasma gets accelerated by this parallel electric field. And most of these parallel accelerated particles are likely lost to the ionosphere (Paschmann et al., 2003); thus they contribute little to the $T_{\mathrm{i}} / T_{\mathrm{e}}$ changes in the magnetosphere.

The contours of the ion-to-electron temperature ratio on the equatorial plane are dawn-dusk symmetric since we did not include the energetic particles' gradient and curvature drifts in the calculation. The finite cross-tail width of the plasma sheet and particle gradient and curvature drift cause depletions of energetic ions on the dawn magnetopause and electrons on the dusk magnetopause, which is likely the main reason for the observed asymmetry of $T_{\mathrm{i}} / T_{\mathrm{e}}$ ratios (i.e., higher $T_{\mathrm{i}} / T_{\mathrm{e}}$ ratios on the duskside). Observations have suggested a dawn-dusk asymmetry, with reconnection occurring more often on the dusk side (e.g., Raj et al., 2002; Kiehas et al., 2018). This asymmetry is not considered in our current model. Since our model shows that reconnection would lead to a lower $T_{\mathrm{i}} / T_{\mathrm{e}}$ ratio, such a dawn-dusk asymmetric reconnection occurrence frequency is expected to reduce the dawn- dusk asymmetry caused by particle gradient and curvature drift. It is noteworthy that particle gradient and curvature drift may violate the conservation of total entropy as plasma moves earthward.

We consider only the earthward side of the half-way cut flux tube since it would eventually stop. On the other hand, the tailward side of the half-way cut flux tube would move tailward and would not stop within the domain we consider. Figures 3 and 4 are of statistical meaning; one would never at a real time (one moment) observe in the magnetosphere a distribution of $T_{\mathrm{i}} / T_{\mathrm{e}}$ ratios such as presented in Figure 4. Figure 4 presents observations averaged over a long period. Figure 3 is a synthesized plot made from the Tsyganenko T96 magnetospheric magnetic field model and the Tsyganenko tail plasma sheet model. The points in Figures 3 and 4 have no physical relation.

It is noteworthy to point out that the Speiser orbit is a special type of non-adiabatic trajectory in the current sheet. Some particles can remain meandering for a time interval very different from the half-gyroperiod (such as those following ring-type orbits, which can meander forever within the current sheet). As stated by Büchner and Zelenyi (1989), ring-type orbits occupy only a small portion (about one tenth) of the phase space; thus we did not include the effect of ring-type orbits in our calculation.

In conclusion, by ignoring the particle's gradient curvature drift and possible precipitation into the ionosphere, and by focusing on the electric field, we demonstrated that patchy magnetic reconnection may preserve the ion-to-electron temperature ratio if charged particles are non-adiabatically accelerated no more than once in each reconnection, while multiple occurrences of nonadiabatic acceleration will cause the ration to vary. We conclude that the observed lower $T_{\mathrm{i}} / T_{\mathrm{e}}$ ratio near the Earth is therefore caused by electrons reflected back from the ionosphere and undergoing multiple non-adiabatic accelerations.

\section{Acknowledgments}

The work by C. X. Chen was supported by the National Nature Science Foundation of China (Grant NSFC41374179). The work by C.P. Wang was supported by NASA (NNX16AJ83G). THEMIS data are available for free with the Space Physics Environment Data Analysis System (SPEDAS) software (http://themis.igpp.ucla.edu/software.shtml). We acknowledge NASA contract NAS5-02099 for THE-MIS, and C. W. Carlson and J. P. McFadden for the use of ESA data, D. Larson and R. P. Lin for use of the SST data.

\section{References}

Angelopoulos, V., Kennel, C. F., Coroniti, F. V., Pellat, R., Kivelson, M. G., Walker, R. J., Russell, C. T., Baumjohann, W., Feldman, W. C., and Gosling, J. T. (1994). Statistical characteristics of bursty bulk flow events. J. Geophys. Res. Space Phys., 99(A11), 21257-21280. https://doi.org/10.1029/94JA01263

Borovsky, J. E., Thomsen, M. F., Elphic, R. C., Cayton, T. E., and McComas, D. J. (1998). The transport of plasma sheet material from the distant tail to geosynchronous orbit. J. Geophys. Res. Space Phys., 103(A9), 20297-20331. https://doi.org/10.1029/97JA03144

Büchner, J., and Zelenyi, L. M. (1989). Regular and chaotic charged particle motion in magnetotaillike field reversals: 1 . Basic theory of trapped motion. J. Geophys. Res. Space Phys., 94(A9), 11821-11842.

https://doi.org/10.1029/JA094iA09p11821

Chen, C. X., and Wolf, R. A. (1999). Theory of thin-filament motion in Earth's 
magnetotail and its application to bursty bulk flows. J. Geophys. Res. Space Phys., 104(A7), 14613-14626. https://doi.org/10.1029/1999JA900005

Chen, C. X. (2013). Theoretical constraints on the cross-tail width of bursty bulk flows. Ann. Geophys., 31(12), 2179-2192. https://doi.org/10.5194/angeo-312179-2013

Chen, C. X. (2016). Substorm onset: A switch on the sequence of transport from decreasing entropy to increasing entropy. Geophys. Res. Lett., 43(10), 4834-4840. https://doi.org/10.1002/2016GL069114

Hardy, D. A., Gussenhoven, M. S., and Brautigam, D. (1989). A statistical model of auroral ion precipitation. J. Geophys. Res. Space Phys., 94(A1), 370-392. https://doi.org/10.1029/JA094iA01p00370

Hill, T. W. (1975). Magnetic merging in a collisionless plasma. J. Geophys. Res., 80(34), 4689-4699. https://doi.org/10.1029/JA080i034p04689

Kiehas, S. A., Runov, A., Angelopoulos, V., Hietala, H., and Korovinksiy, D. (2018). Magnetotail fast flow occurrence rate and dawn-dusk asymmetry at $X_{\mathrm{GMS}}$ $-60 R_{\mathrm{E}}$. J. Geophys. Res. Space Phys., 123(3), 1767-1778. https://doi.org/10.1002/2017JA024776

Nakamura, R., Baumjohann, W., Mouikis, C., Kistler, L. M., Runov, A., Volwerk, M., Asano, Y., Vörös, Z., Zhang, T. L., ... Balogh, A. (2004). Spatial scale of highspeed flows in the plasma sheet observed by Cluster. Geophys. Res. Lett., 31(9), L09804. https://doi.org/10.1029/2004GL019558

Onsager, T. G., Thomsen, M. F., Elphic, R. C., and Gosling, J. T. (1991). Model of electron and ion distributions in the plasma sheet boundary layer. J. Geophys. Res. Space Phys., 96(A12), 20999-21011. https://doi.org/10.1029/91JA01983

Onsager, T. G., Scudder, J. D., Lockwood, M., and Russell, C. T. (2001). Reconnection at the high-latitude magnetopause during northward interplanetary magnetic field conditions. J. Geophys. Res. Space Phys., 106(A11), 25467-25488. https://doi.org/10.1029/2000JA000444

Paschmann, G., Haaland, S., and Treumann, R. (2003). In situ measurements in the auroral plasma. In G. Paschmann, et al. (Eds.), Auroral Plasma Physics (pp. 93-208). Dordrecht, Netherlands: Springer. https://doi.org/10.1007/978-94007-1086-3

Raj, A., Phan, T., Lin, R. P., and Angelopoulos, V. (2002). Wind survey of highspeed bulk flows and field-aligned beams in the near-Earth plasma sheet. $J$. Geophys. Res. Space Phys., 107(A12), SMP 3-1-SMP 3-17. https://doi.org/10.1029/2001JA007547
Rijnbeek, R. P., Cowley, S. W. H., Southwood, D. J., and Russell, C. T. (1984). A survey of dayside flux transfer events observed by ISEE 1 and 2 magnetometers. J. Geophys. Res. Space Phys., 89(A2), 786-800. https://doi.org/10.1029/JA089iA02p00786

Schriver, D., Ashour-Abdalla, M., and Richard, R. L. (1998). On the origin of the ion-electron temperature difference in the plasma sheet. J. Geophys. Res. Space Phys., 103(A7), 14879-14895. https://doi.org/10.1029/98JA00017

Slavin, J. A., Smith, E. J., Sibeck, D. G., Baker, D. N., Zwickl, R. D., and Akasofu, S. I. (1985). An ISEE 3 study of average and substorm conditions in the distant magnetotail. J. Geophys. Res. Space Phys., 90(A11), 10875-10895. https://doi.org/10.1029/JA090iA11p10875

Speiser, T. W. (1965). Particle trajectories in model current sheets: 1. Analytical solutions. J. Geophys. Res., 70(17), 4219-4226. https://doi.org/10.1029/JZ070i017p04219

Tsyganenko, N. A. (1995). Modeling the Earth's magnetospheric magnetic field confined within a realistic magnetopause. J. Geophys. Res. Space Phys., 100(A4), 5599-5612. https://doi.org/10.1029/94JA03193

Tsyganenko, N. A., and Mukai, T. (2003). Tail plasma sheet models derived from Geotail particle data. J. Geophys. Res. Space Phys., 108(A3), 1136. https://doi.org/10.1029/2002JA009707

Wang, C. P., Lyons, L. R., Wolf, R. A., Nagai, T., Weygand, J. M., and Lui, A. T. Y. (2009). The plasma sheet $p V^{5 / 3}$ and $n v$ and associated plasma and energy transport for different convection strengths and $A E$ levels. J. Geophys. Res. Space Phys., 114(A9), A00D02. https://doi.org/10.1029/2008JA013849

Wang, C. P., Gkioulidou, M., Lyons, L. R., and Angelopoulos, V. (2012). Spatial distributions of the ion to electron temperature ratio in the magnetosheath and plasma sheet. J. Geophys. Res. Space Phys., 117(A8), A08215. https://doi.org/10.1029/2012JA017658

Wing, S., Johnson, J. R., Chaston, C. C., Echim, M., Escoubet, C. P., Lavraud, B., Lemon, C., Nykyri, K., Otto, A., ... Wang, C. P. (2014). Review of solar wind entry into and transport within the plasma sheet. Space Sci. Rev., 184(1-4), 33-86. https://doi.org/10.1007/s11214-014-0108-9

Zesta, E., Donovan, E., Lyons, L., Enno, G., Murphree, J. S., and Cogger, L. (2002). Two-dimensional structure of auroral poleward boundary intensifications. $J$. Geophys. Res. Space Phys., 107(A11), SIA 6-1-SIA 6-20. https://doi.org/10.1029/2001JA000260 